\title{
Massive Stars at High Redshifts
}

\author{
Max Pettini \\ Institute of Astronomy, University of Cambridge, Cambridge, CB3 0HA, UK
}

\begin{abstract}
The five years that have passed since the last IAU Symposium devoted to massive stars have seen a veritable explosion of data on the high redshift universe. The tools developed to study massive stars in nearby galaxies are finding increasing application to the analysis of the spectra of star-forming regions at redshifts as high as $z=7$. In this brief review, I consider three topics of relevance to this symposium: the determination of the metallicities of galaxies at high redshifts from consideration of their ultraviolet stellar spectra; constraints on the initial mass function of massive stars in galaxies at $z=2-3$; and new clues to the nucleosynthesis of carbon and nitrogen in massive stars of low metallicity. The review concludes with a look ahead at some of the questions that may occupy us for the next five years (at least!).
\end{abstract}

Keywords. galaxies: abundances - quasars: absorption lines - stars: abundances - stars: WolfRayet

\section{Introduction}

The fact that a whole session of IAU Symposium 250 is devoted to the high redshift universe is an eloquent demonstration of how much our observational capabilities have improved in recent years. When we record the spectra of galaxies at redshifts $z>1$ with our ground-based optical and infrared telescopes we of course look directly at their rest-frame ultraviolet (UV) emission that is dominated by massive stars. Thus, many of the ideas and techniques which we have discussed in the last four days find immediate application at high redshift, thereby reinforcing the already strong link between the stellar and extragalactic communities of astronomers.

In the last five years, since the last IAU Symposium devoted to massive stars in Lanzarote, studies of high redshift galaxies have proliferated. A number of different large-scale surveys have given us samples of hundreds or thousands of galaxies at redshifts from zero all the way to the end of the 'Dark Ages' at $z \sim 7$. A clear illustration of such progress is provided by Figure 1. The small size of the error bars in each bin of the luminosity functions attests to the improvements in the number statistics afforded by the large scale of galaxy surveys.

Out of this considerable body of recent work on high redshift galaxies I shall consider three main topics of particular relevance to this meeting: (i) determinations of element abundances; (ii) the initial mass function of massive stars; and (iii) new clues to the nucleosynthesis of $\mathrm{C}, \mathrm{N}$, and $\mathrm{O}$ in the first few episodes of star formation.

\section{Abundance Determinations in High Redshift Galaxies}

The degree to which galaxies at high redshifts have processed their interstellar media into stars, and the attendant detailed pattern of element abundances, are key physical characteristics that give us the means to assess their evolutionary status and link them to today's galaxy populations. To this end, a great deal of effort has been devoted to developing different metallicity diagnostics applicable at high redshifts - a review is given by Pettini (2006). 


\section{Luminosity Function}
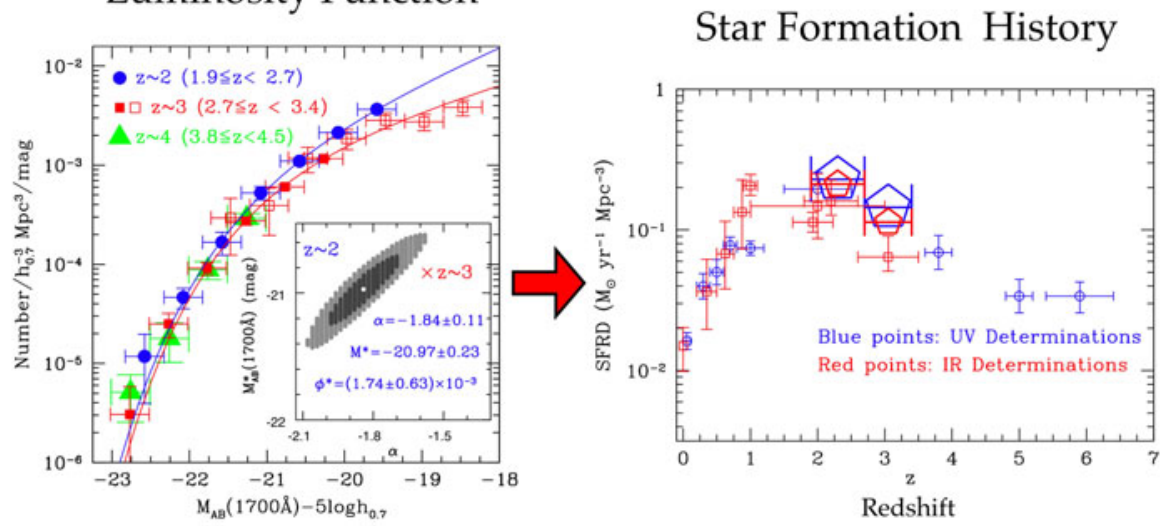

Figure 1. Figures reproduced from Reddy et al. (2008) showing the most recent and comprehensive determinations of: (left) the galaxy luminosity function at redshifts $z=2-4$, and (right) the redshift evolution of the comoving star formation rate density.

Metallicities of galaxies at $z>1$ have been deduced from consideration of (i) nebular emission lines formed in ionised gas at the sites of star formation; (ii) interstellar absorption lines which probe the more widely distributed interstellar medium; and (iii) spectral features from the photospheres and winds of massive stars. It is reassuring that these different approaches give concordant answers, to within a factor of about two, in the few cases where it has so far been possible to cross-check them in the same galaxy.

Of these methods, the one which has been found the widest application up to now is the first. For practical reasons, the ratio of two closely spaced emission lines, $\mathrm{H} \alpha$ and [N II] 26583 , (Pettini \& Pagel 2004) has proved to be particularly useful for obtaining a first, approximate measure of the abundance of oxygen in galaxies at $z \simeq 2$. In conjunction with estimates of the assembled stellar mass deduced from consideration of the restframe ultraviolet to near-infrared spectral energy distribution, this has led to the first determination of a mass-metallicity relation in galaxies at these redshifts, illustrated in Figure 2 .

A general conclusion seems to be that UV-bright, star-forming, galaxies at $z \simeq 2$ were already at an advanced stage of chemical evolution: $85 \%$ of the galaxies in the Erb et al. (2006) sample have an oxygen abundance greater than, or equal to, $2 / 5$ of that of the Orion nebula. There appears to have been only mild redshift evolution in the massmetallicity relation over the last $3 / 4$ of the age of the universe: some 10 billion years ago, galaxies of a given stellar mass were less metal-rich by only a factor of about two compared to the galaxies around us today as mapped by the Sloan Digital Sky Survey.

One of the difficulties in deriving abundances from nebular emission lines is that the strongest and best calibrated features all occur at rest-frame optical wavelengths which are redshifted into the near-infrared at $z>1$, a wavelength regime where observations are complicated by a high (and variable) sky background and by the lack (until now) of multi-object spectrographs. There is therefore a strong incentive to try and develop abundance measures based on the spectral features from massive stars which dominate the rest-frame ultraviolet spectra of high- $z$ galaxies - readily accessible from the ground at redshifts $z>1$. This is at the approach adopted by Rix et al. (2004), partly stimulated by discussions at the Lanzarote meeting five years ago (see Figure 3). 


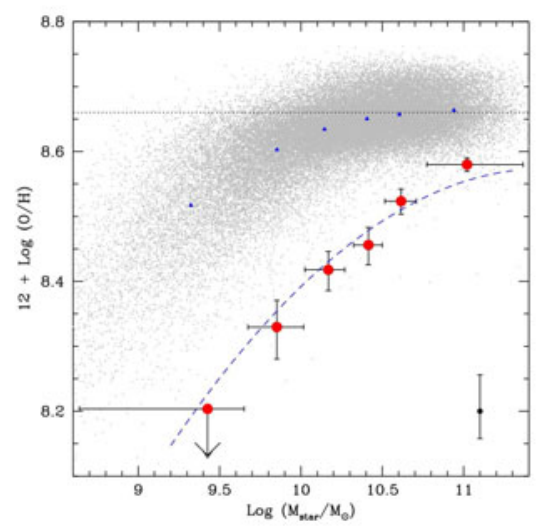

Figure 2. Stellar mass-metallicity relation for star-forming galaxies at $z \sim 2$ (large red circles with error bars) and in the nearby $(z \sim 0.1)$ universe from the SDSS survey (small grey points). The blue triangles show the mean metallicity of the SDSS galaxies in the same mass bins as the $z=2$ galaxies. The vertical error bar in the bottom right-hand corner indicates the uncertainty in the calibration of the $N 2$ index (Pettini \& Pagel 2004) used to deduce the oxygen abundance of both sets of galaxies. The horizontal dotted line is drawn at the solar abundance of oxygen. (Figure reproduced from Erb et al. 2006).

As pointed out by Leitherer et al. (2001), the spectral synthesis code Starburst99 provides the machinery required to assess which stellar spectral features are most sensitive to changes in metallicity. However, its usefulness in this respect is limited by the lack of observations of $\mathrm{OB}$ stars of different metallicities - the currently available empirical libraries come in only two flavours: 'Milky Way' and 'Magellanic Cloud' (a hybrid of Large and Small Magellanic Cloud stars). To overcome this problem, Rix et al. (2004) turned to the fully theoretical massive star spectra generated by the $W$ - $M$ Basic code developed in Munich by Pauldrach et al. (2001) and used them to synthesise fully theoretical composite spectra of star-forming galaxies spanning a wide range of metallicities. Some examples are reproduced in Figure 4.

This approach turned out to be very successful. The $W$-M Basic galaxy spectra match closely those generated with the empirical libraries at the metallicities and wavelengths common to the two versions of Starburst99, giving us confidence in the extrapolations to

Model Starburst Spectrum WM-Basic+Starburst99 (Rix et al. 2004)

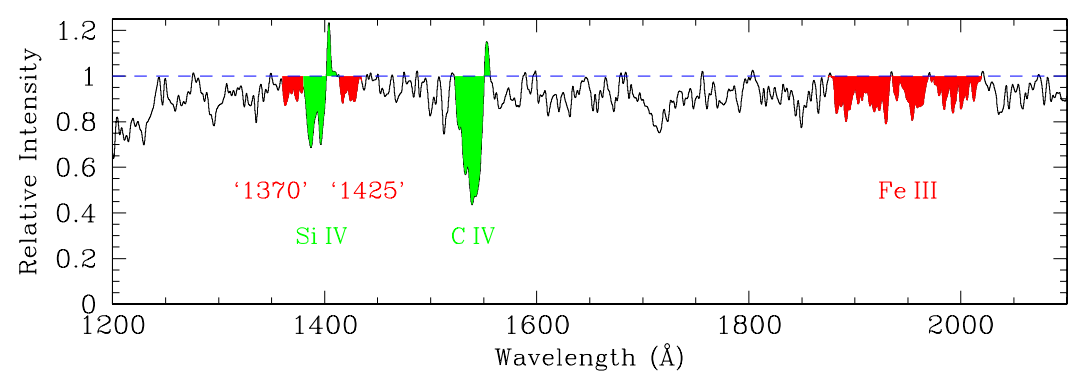

Figure 3. Fully theoretical UV spectrum of a starburst galaxy computed by Rix et al. (2004) for the case of continuous star formation with a Salpeter initial mass function (IMF) and solar metallicity. The shaded regions indicate stellar wind lines (green) and blends of photospheric lines (red) found by Rix et al. to be sensitive to metallicity. 


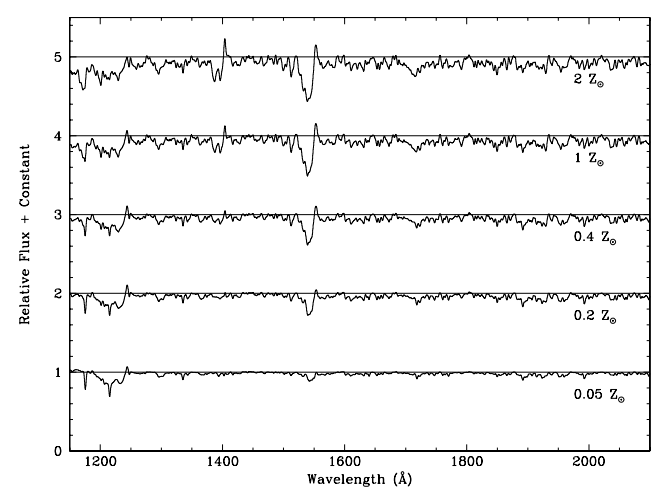

Figure 4. Fully synthetic UV spectra of star-forming galaxies with metallicities $Z=2-0.05 \mathrm{Z}_{\odot}$, from the work by Rix et al. (2004). All the examples shown here are for the 'standard' case of continuous star formation (at age $100 \mathrm{Myr}$ ) with a Salpeter slope of the IMF between 1 and $100 \mathrm{M}_{\odot}$. The spectra have been convolved with a Gaussian of FWHM $=2.5 \AA$ (to match the typical rest-frame resolution of observed spectra of high- $z$ galaxies) and normalised to the underlying stellar pseudo-continuum. See Rix et al. (2004) for additional details.

other metallicities and wavelength ranges (the empirical libraries are limited to a rather small portion of the UV spectrum). When compared to the best available spectra of

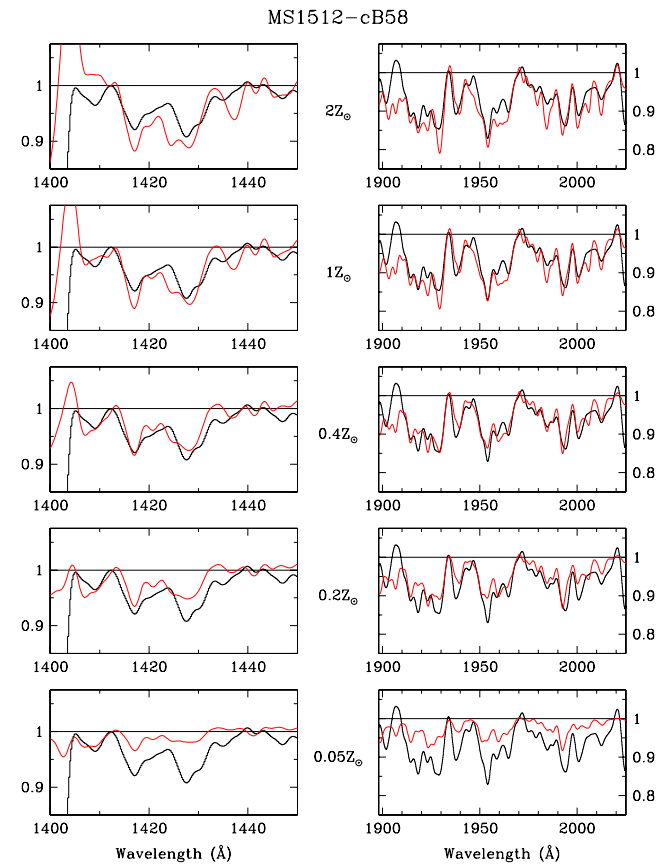

Figure 5. Comparison of the observed spectrum of the $z=2.7276$ Lyman break galaxy MS1512-cB58 (black) with fully synthetic spectra produced by the Starburst99+W-M Basic combination. The left-hand set of panels shows the region near $1425 \AA$ which includes a blend of $\mathrm{Si}, \mathrm{C}$, and $\mathrm{Fe}$ photospheric lines, while the right-hand set is for the blend of Fe III lines near $1978 \AA$ produced in the photospheres of late O- and early B-type stars. The $y$-axis is residual intensity. (Figure reproduced from Rix et al. 2004). 
high- $z$ star-forming galaxies (Figure 5), the $W$-M Basic version of Starburst99 scores very highly, in being able to reproduce the fine detail of blends of photospheric features centred near $1425 \AA$ and $1978 \AA$. As can be seen from Figure 5 , the closest match between model and observed spectra of MS1512-cB58 (a strongly lensed Lyman break galaxy at $z=2.7276$ ) in these wavelength regions is obtained for a stellar metallicity of $\sim 2 / 5$ solar (middle panels). This value is in excellent agreement with the metallicity of the interstellar medium of this galaxy, as determined from absorption lines from H I regions (Pettini et al. 2002) and nebular lines from H II regions (Teplitz et al. 2000).

These results are very encouraging in offering an additional avenue to determining the metallicity of high- $z$ galaxies, particularly at redshifts $z>4$ where other diagnostics may be difficult to apply. On a less optimistic note, it is clear from these initial trials that data of high signal-to-noise ratio are essential in order to correctly interpret these low-contrast blends of stellar photospheric lines (e.g. de Mello et al. 2004). With current instrumentation such high quality data can only be secured for a handful of strongly lensed high redshift galaxies (whose number is now increasing thanks to the Sloan Digital Sky Survey). However, looking further ahead, it is easy to foresee that this technique will be fully exploited with the advent of 30-m class telescopes in the next decade (see Sandro D'Odorico's contribution to this volume).
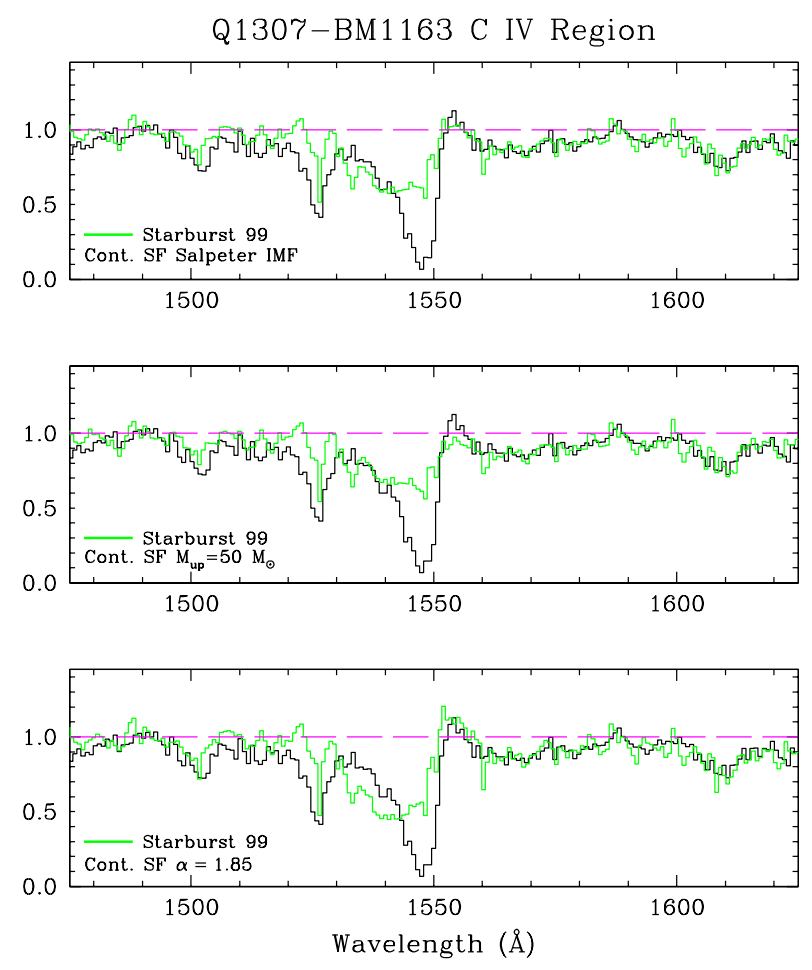

Figure 6. Sensitivity of the CIV P-Cygni emission-absorption profile to the upper end of the IMF. Black histogram: portion of the observed spectrum of Q1307-BM1163, a bright star-forming galaxy at $z=1.409$; green histograms: model spectra produced by the Starburst99 spectral synthesis code with, respectively, a standard Salpeter IMF (top panel), an IMF lacking stars more massive than $50 \mathrm{M}_{\odot}$ (middle panel), and an IMF flatter than Salpeter (bottom panel). The changes were deliberately chosen to be relatively small to illustrate the sensitivity of the UV spectral features to the mix of stellar masses. (Figure reproduced from Steidel et al. 2004). 


\section{The Initial Mass Function in Star-Forming Galaxies at Redshifts $z=2-3$}

Between $\sim 1000$ and $\sim 2000 \AA$, the UV spectrum of a star-forming galaxy is dominated by the light from stars more massive than about $10 \mathrm{M}_{\odot}$. Consequently, the details of such spectra-particularly the contrast of spectral features due to the most massive stars over the underlying integrated continuum - offer a direct test of the IMF of massive stars. A fair assessment of the situation so far would be to say that no evidence has yet been found for departures from a Salpeter slope at the high mass end of the IMF. An illustration is provided in Figure 6 showing a portion of the UV spectrum of the bright $z=1.409$ galaxy Q1307-BM1163 from Steidel et al. (2004). After excluding the narrow interstellar core of the C IV line (Crowther et al. 2006), the best fit to the P-Cygni emission-absorption profile is achieved with a Salpeter IMF from 1 to $120 \mathrm{M}_{\odot}$ (top panel). Even minor changes, such as truncating the IMF at $50 \mathrm{M}_{\odot}$ (middle panel), or increasing the proportion of massive stars by adopting a flatter slope ( $\alpha=1.85$ instead of 2.35 -bottom panel) result in synthetic C IV profiles that are evidently poorer matches to the observations.

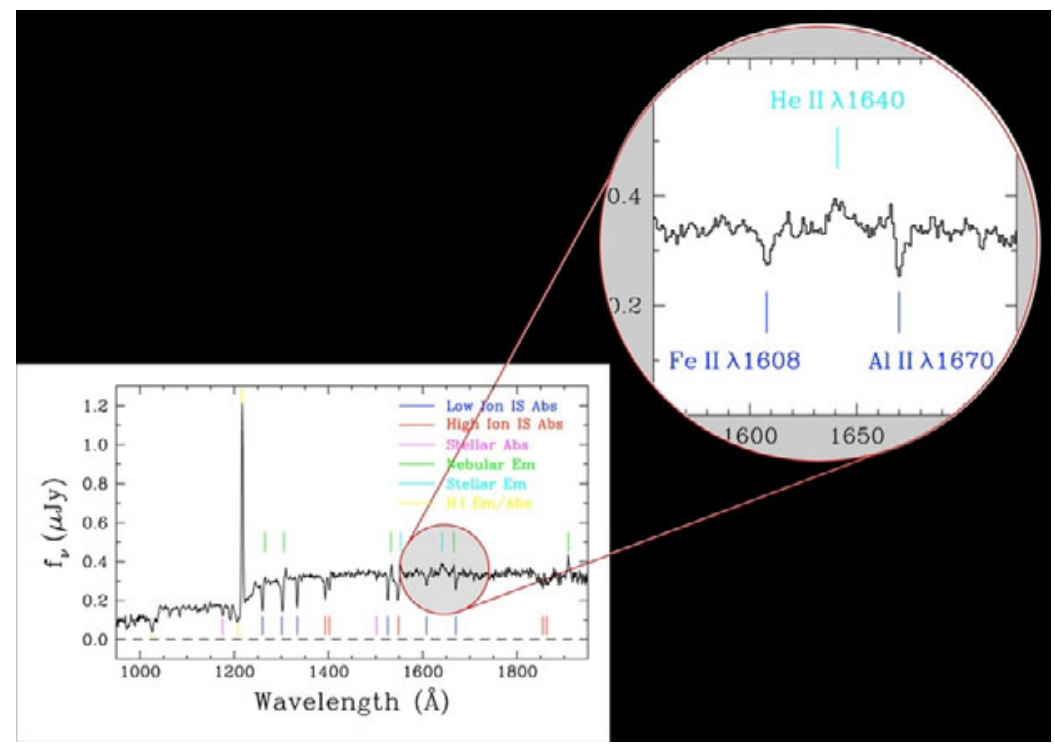

Figure 7. Composite rest-frame UV spectrum of 811 Lyman break galaxies at $\langle z\rangle=3.0 \pm 0.3$ from Shapley et al. (2003). The co-addition of so many spectra reveals the wealth of spectral features available for analysis. The inset shows the broad He II $\lambda 1640$ emission line produced by Wolf-Rayet stars, superimposed on the integrated stellar continuum with an equivalent width $\operatorname{EW}(\lambda 1640)=1.3 \pm 0.3 \AA$.

Another spectral feature that is regularly recognised in the spectra of Lyman break galaxies (at least when recorded at sufficiently high signal-to-noise ratio) is a broad He II $\lambda 1640$ emission line from Wolf-Rayet stars. The ubiquitous presence of this line is demonstrated by the fact that it can be readily discerned in the composite spectrum of $811 z \simeq 3$ galaxies constructed by Shapley et al. (2003- see Figure 7). It has been suggested that a top-heavy IMF, or even a population of primordial stars (Jimenez \& Haiman 2006), may be required to explain the strength of this feature. In reality, the recent analysis by Brinchmann, Pettini, \& Charlot (2008), which uses up-to-date calibrations of the luminosity of this line in W-R stars of different sub-classes and different 


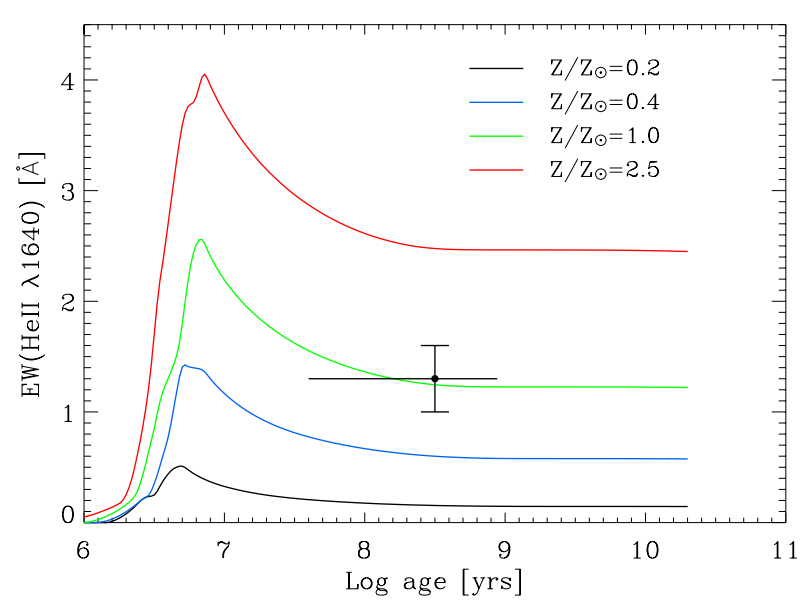

Figure 8. Time evolution of the equivalent width of the He II $\lambda 1640$ emission line for four difference metallicities (from the models by Brinchmann et al. 2008). All four curves refer to a model with a constant star formation rate and Salpeter IMF. The dot at Log age [yrs] $=8.5$ is the value of $\mathrm{EW}$ (He II $\lambda 1640$ ) measured from the composite spectrum of 811 Lyman break galaxies published by Shapley et al. (2003 - see Figure 7), while the error bar shows the inter-quartile range of their ages (Shapley et al. 2001). The measured value of $\mathrm{EW}(\mathrm{He}$ II $\lambda 1640$ ) is reproduced by the models with metallicities $Z=0.75-1.5 \mathrm{Z}_{\odot}$, somewhat higher than, but still consistent with, current estimates of the metallicity of LBGs.

metallicities (see the contributions to this volume by Paul Crowther and Lucy Hadfield), has shown that the observed $\operatorname{EW}(\lambda 1640)=1.3 \pm 0.3 \AA$ in the composite in Figure 7 can be naturally understood in terms of a Salpeter IMF and metallicities typical of LBGs, i.e. $Z_{\mathrm{LBG}} \simeq 0.3-1 \mathrm{Z}_{\odot}$, as in Figure 2 (see Figure 8 ).

\section{C, N, O Nucleosynthesis by Metal-Poor Stars}

One of the topics of discussion at this meeting has been the recently recognised importance of massive stars to the nucleosynthesis not only of oxygen, but also of carbon and nitrogen, in the low metallicity regime (see the contributions to this volume by Raphael Hirschi, André Maeder, and Georges Meynet). Theoretical efforts to calculate the yields of these elements as a function of metallicity in stellar models that include the effects of rotation have been motivated by recent observations of elevated ratios of $\mathrm{C} / \mathrm{O}$ and $\mathrm{N} / \mathrm{O}$ in some of the most metal-poor stars in the halo of the Milky Way (Akerman et al. 2004; Spite et al. 2005).

Damped Ly $\alpha$ systems, or DLAs, (a class of absorption line systems in the spectra of high redshift quasars distinguished by their high column densities of neutral hydrogen) offer a complementary way to verify such trends in the relative abundances of $\mathrm{C}, \mathrm{N}$, and $\mathrm{O}$ (Pettini, Lipman, \& Hunstead 1995). The measurements of element abundances are often more straightforward from the interstellar lines in DLAs than from the photospheres of halo stars, requiring fewer assumptions about the physical conditions and geometry of the gas in which they are formed. The main limitation is the restricted dynamic range of interstellar absorption lines - once a line becomes saturated it is no longer useful for abundance determinations. 

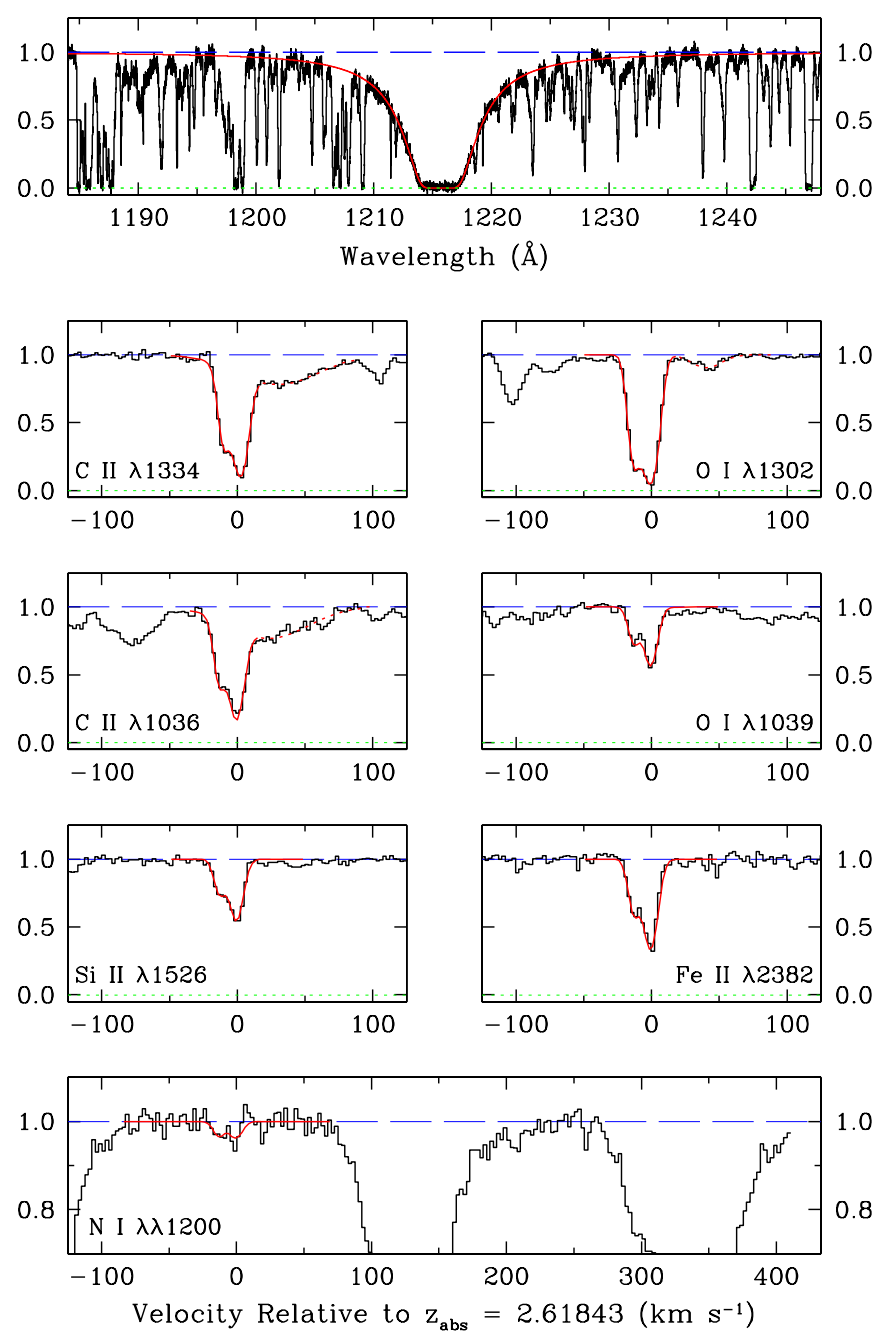

Figure 9. Observed profiles (black histograms) and fitted Voigt profiles (continuous red lines) of selected absorption lines in the $z_{\mathrm{abs}}=2.61843$ DLA in the QSO Q0913+072. The $y$-axes of the plots show residual intensity; note the expanded $y$-scale of the bottom plot. The top panel shows the damped Ly $\alpha$ line, indicative of a column density of neutral hydrogen $N(\mathrm{H} \mathrm{I})=2.2 \times 10^{20}$ atoms $\mathrm{cm}^{-2}$. With an oxygen abundance of only $1 / 250$ of solar, this is one of the most metal-poor DLAs known. The data shown here were obtained with UVES on the VLT and are reproduced from Pettini et al. (2008).

\subsection{Carbon}

The problem of line saturation has been particularly acute for $\mathrm{C}$ : the high abundance of this element (the fourth most abundant in the periodic table after $\mathrm{H}, \mathrm{He}$, and $\mathrm{O}$ ), combined with the high transition probabilities of the resonance lines of its first ion (its dominant ion stage in $\mathrm{H}$ I regions), has been an obstacle to a precise determination of the interstellar abundance of $\mathrm{C}$ even in the nearby interstellar medium (e.g. Hobbs et al. 1982).

Recently, Pettini et al. (2008) have attempted to circumvent these difficulties by searching for the most metal-poor DLAs in the growing sample of such absorbers discovered by 


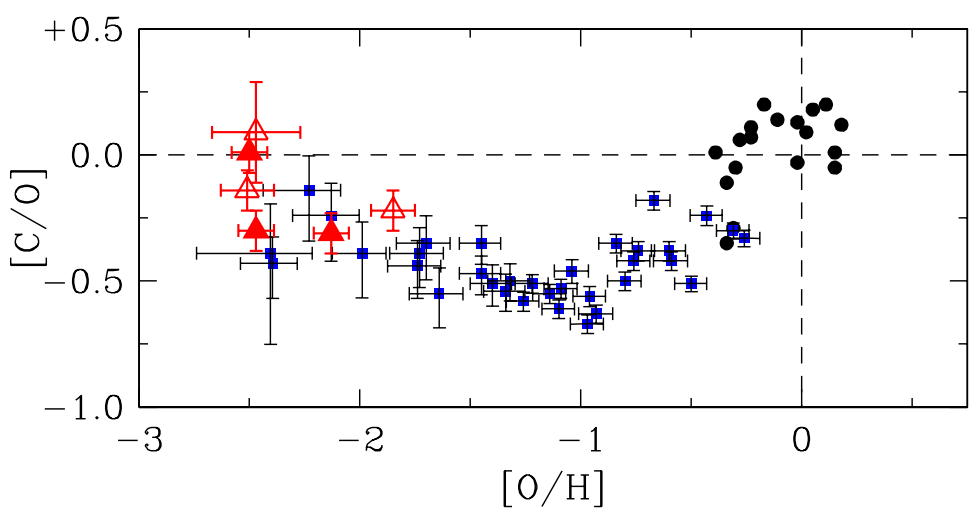

Figure 10. The values of $[\mathrm{C} / \mathrm{O}]$ deduced by Pettini et al. (2008) for DLAs (red filled triangles) and sub-DLAs (open triangles) broadly agree with the stellar abundances (dots) measured at similar metallicities, supporting the suggestion by Akerman et al. (2004) that carbon and oxygen may be produced in near-solar proportions in the earliest stages of galactic chemical evolution. Stellar data from Akerman et al. (2004) and references therein (black dots: disk stars; blue squares: halo stars).

Prochaska, Herbert-Fort, \& Wolfe (2005) in the spectra of QSOs from the Sloan Digital Sky Survey. An example is reproduced in Figure 9. At metallicities $Z_{\mathrm{DLA}}<1 / 100 \mathrm{Z}_{\odot}$, the resonance lines of $\mathrm{C}$ II are only mildly saturated and the velocity structure of the absorbing gas is generally simple, allowing for a more straightforward determination of the $\mathrm{C} / \mathrm{O}$ and $\mathrm{O} / \mathrm{H}$ ratios.

Figure 10 shows $[\mathrm{C} / \mathrm{O}]$ vs. $[\mathrm{O} / \mathrm{H}]$ in Galactic stars and the few DLAs in which the abundances of $\mathrm{C}$ and $\mathrm{O}$ have been measured to date; as usual, the square bracket notation denotes logarithmic abundances relative to the solar values (Asplund, Grevesse, \& Sauval $2005)$. In halo stars $[\mathrm{C} / \mathrm{O}] \approx-0.5$, while at the higher metallicities of most disk stars $[\mathrm{C} / \mathrm{O}]$ rises to solar proportions. In the Galactic chemical evolution models considered by Akerman et al. (2004) this rise is interpreted as the additional contribution to carbon enrichment of the interstellar medium from the winds of Wolf-Rayet stars - whose mass loss rates are known to increase with metallicity — and, to a lesser extent, from the delayed evolution of low and intermediate mass stars.

More puzzling is the apparent upturn in $[\mathrm{C} / \mathrm{O}]$ with decreasing metallicity when $[\mathrm{O} / \mathrm{H}] \lesssim-1$, since conventional stellar yields would predict the opposite behaviour: the expected time lag in the production of $\mathrm{C}$ relative to $\mathrm{O}$ in the first episodes of star formation should cause the $[\mathrm{C} / \mathrm{O}]$ ratio to decrease dramatically as we move to the lowest metallicities in Figure 10. Akerman et al. (2004) speculated that the trend towards higher, rather than lower, values of $[\mathrm{C} / \mathrm{O}]$ when $[\mathrm{O} / \mathrm{H}]<-1$ may be due to remaining traces of high carbon production by the first stars to form in the halo of the Milky Way proto-galaxy - high carbon yields (relative to oxygen) are indeed predicted by calculations of nucleosynthesis by Population III stars (e.g. Chieffi \& Limongi 2002). More recently, Chiappini et al. (2006 - see also Georges Meynet's and Raphael Hirschi's contributions to this volume) proposed that the observed behaviour can also be explained if stars of lower metallicity rotate faster: the higher rotation speeds greatly increase the yields of $\mathrm{C}$ (and $\mathrm{N}$ ) by the massive stars which are the main source of $\mathrm{O}$.

Some uncertainty in the interpretation of the stellar data is the use by Akerman et al. (2004) of one-dimensional, local thermodynamical equilibrium (LTE), stellar atmosphere models. The authors pointed out themselves the possibility that the neglect of non-LTE 
corrections to the high excitation $\mathrm{C}$ I and $\mathrm{O}$ I lines used for abundance determinations may mimic (or at least enhance) the rise in $[\mathrm{C} / \mathrm{O}]$ with decreasing $[\mathrm{O} / \mathrm{H}]$. However, when the values of $[\mathrm{C} / \mathrm{O}]$ measured in DLAs are compared with those of Galactic stars as in Figure 10, it appears that both sets of data paint a consistent picture.

The DLA measurements corroborate the conclusion by Akerman et al. (2004) in showing relatively high values of $\mathrm{C} / \mathrm{O}$ in the low metallicity regime and complement the still very limited statistics of this ratio in stars of metallicity $[\mathrm{O} / \mathrm{H}]<-2$. It must be borne in mind here that, although the high redshift DLAs and the halo stars appear to constitute a continuous sequence in Figure 10, this does not imply that the chemical enrichment histories of the Milky Way halo and of the galaxies giving rise to DLAs were the same. It may well be that, as chemical evolution progresses, different DLAs follow different paths in the $[\mathrm{C} / \mathrm{O}]$ vs. $[\mathrm{O} / \mathrm{H}]$ plane, some similar to that of the Milky Way stellar populations while others may be different. Such a variety of behaviours has already been seen (in other elements) in Local Group galaxies (e.g. Pritzl et al. 2005). What our data show is that at metallicities below 1/100 of solar, DLAs and Galactic stars concur in showing elevated ratios of $\mathrm{C} / \mathrm{O}$. Akerman et al. (2004) speculated that an approximately solar value of $\mathrm{C} / \mathrm{O}$ might be recovered when $[\mathrm{O} / \mathrm{H}] \lesssim-3$; the new data presented here are certainly consistent with this extrapolation of the Galactic measurements.

\subsection{Nitrogen}

The metal-poor DLAs targeted by Pettini et al. (2008) also help assess the contribution (if any) by massive stars to the primary production of $\mathrm{N}$. The dual channels for the nucleosynthesis of N ('primary' or 'secondary', depending on whether the seed carbon and oxygen required for the CNO cycle are those manufactured by the star during helium burning, or were already present when the star first condensed out of the interstellar medium) are revealed by plots of $\mathrm{N} / \mathrm{O}$ vs. $\mathrm{O} / \mathrm{H}$ in extragalactic $\mathrm{H}$ II regions, such as the one reproduced in Figure 11. When the oxygen abundance is greater than about half solar (i.e. when $\log (\mathrm{O} / \mathrm{H})+12 \gtrsim 8.3) \mathrm{N} / \mathrm{O}$ rises steeply with increasing $\mathrm{O} / \mathrm{H}$; this is the regime where $\mathrm{N}$ is predominantly secondary. At lower metallicities on the other hand $(\log (\mathrm{O} / \mathrm{H})+12 \lesssim 8.0), \mathrm{N}$ is mostly primary and its abundance tracks that of $\mathrm{O}$; this results in the flat plateau at $\log (\mathrm{N} / \mathrm{O}) \simeq-1.5$ evident in Figure 11 .

Primary $\mathrm{N}$ is thought to be synthesised most effectively by intermediate mass stars on the asymptotic giant branch; consequently, its release into the interstellar medium presumably takes place some time after the massive stars that are the main source of oxygen have exploded as Type II supernovae. Henry, Edmunds, \& Köppen (2000) calculated the delay to be $\Delta t \sim 250 \mathrm{Myr}$ for a 'standard' stellar initial mass function (IMF) and published stellar yields.

Damped Ly $\alpha$ systems add information that can be of value in assessing the validity of this overall picture. First of all, they offer measures of the abundances of $\mathrm{O}$ and $\mathrm{N}$ in entirely different environments from nearby star-forming galaxies. Second, they sample low metallicity regimes which are rare today. And third, by virtue of their being at high redshift, they can in principle provide a better sampling of the time delay in the production of primary $\mathrm{N}$ relative to $\mathrm{O}$ - the canonical $\Delta t \sim 250 \mathrm{Myr}$ is a much larger fraction of the time available for star formation at $z=2-3$ than today. In other words, the chances of 'catching' a galaxy in the interim period following a burst of star formation, when the $\mathrm{O}$ from Type II supernovae has already dispersed into the interstellar medium but lower mass stars have yet to release their primary $\mathrm{N}$, are considerably higher in DLAs than in metal-poor star-forming galaxies in the local universe. Such a situation should manifest itself as a displacement below the primary plateau in the $\log (\mathrm{N} / \mathrm{O})$ vs. $\log (\mathrm{O} / \mathrm{H})$ plane in Figure 11. 


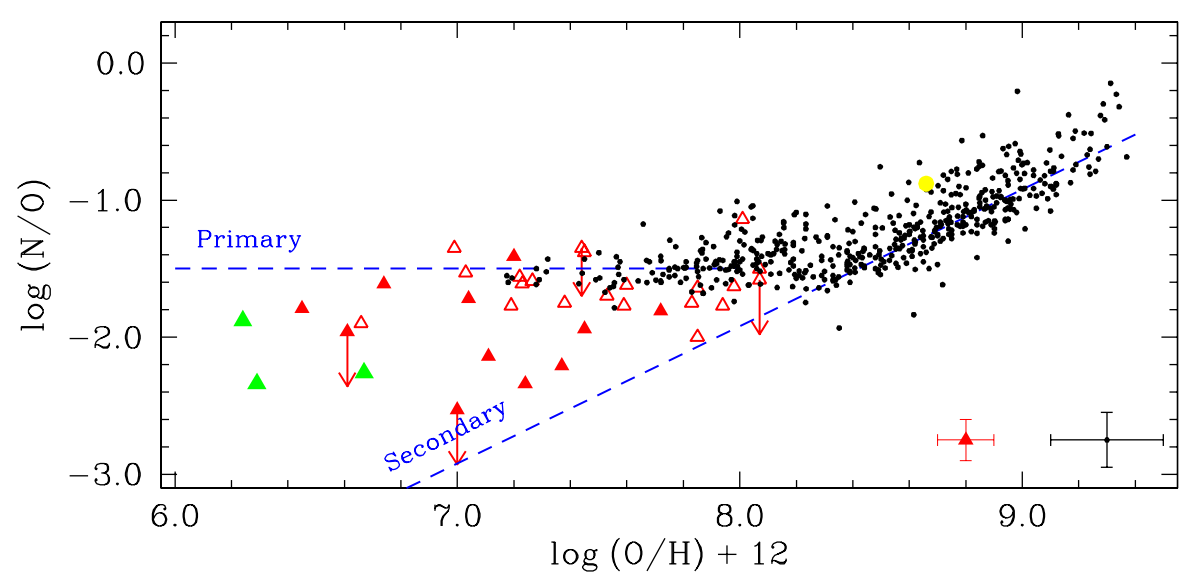

Figure 11. Abundances of $\mathrm{N}$ and $\mathrm{O}$ in extragalactic $\mathrm{H}$ II regions (small dots) and high redshift damped Ly $\alpha$ systems (triangles). See Pettini et al. (2008) for the sources of the original measurements. Filled triangles denote DLAs where the abundance of O could be measured directly, while open triangles are cases where $\mathrm{S}$ was used as a proxy for $\mathrm{O}$. The error bars in the bottom right-hand corner give an indication of the typical uncertainties; the large yellow dot corresponds to the solar abundances of $\mathrm{N}$ and $\mathrm{O}$. The dashed lines are approximate representations of the primary and secondary levels of $\mathrm{N}$ production (see text).

When DLAs are compared with present-day galaxies, as in Figure 11, it can be seen that in most cases their values of $\mathrm{N} / \mathrm{O}$ fall close to the plateau defined by metal-poor $\mathrm{H}$ II regions. If primary $\mathrm{N}$ and $\mathrm{O}$ are synthesised by stars of different masses, as generally believed, the good agreement between local and distant galaxies attests to the universality of the IMF - a top-heavy IMF in DLAs would result in lower values of N/O, contrary to observations.

The finding that $\sim 1 / 3$ of DLAs in the current sample have values of $N / O$ lower than the primary plateau is consistent with current ideas of a time delay by a few $10^{8}$ years in the release of primary $\mathrm{N}$ from intermediate mass stars. But Figure 11 also emphasises the importance of extending measurements of $\mathrm{N} / \mathrm{O}$ to the lowest metallicity DLAs: as the gap between secondary and primary $\mathrm{N}$ production widens, it becomes easier to recognise additional sources of primary nitrogen, if they exist. In particular, if massive stars were able to synthesise some primary $\mathrm{N}$, we might expect to see a minimum value of $\mathrm{N} / \mathrm{O}$, corresponding to the $\mathrm{N}$ ejected into the ISM at the same time as O (Centurión et al. 2003). While the statistics are still limited, the available data do hint at the possibility of a 'floor' in the value of $\mathrm{N} / \mathrm{O}$ at $\log (\mathrm{N} / \mathrm{O}) \simeq-2.3$. If this does indeed reflect the primary $\mathrm{N}$ yield by massive stars, it would imply that at these metallicities massive stars can account for $\sim 15 \%$ of the total primary $\mathrm{N}$ production.

We have heard much at this meeting about the importance of stellar rotation in facilitating the nucleosynthesis of primary $\mathrm{N}$ in massive stars (Hirschi et al. 2007-see also Georges Meynet's and Raphael Hirschi's contributions to this volume). It is therefore worthwhile pointing out here that the level of primary $\mathrm{N}$ production by massive stars suggested by the DLA measurements is lower than that apparently implied by the generally higher values of N/O derived by Spite et al. (2005) in metal-poor halo stars. It is the latter that has motivated much of the theoretical nucleosynthesis calculations so far. Unfortunately, the derivation of $\mathrm{N}$ abundances in very metal-poor stars remains one of the most difficult problems in stellar abundance measurements, beset with systematic 
uncertainties as Spite et al. (2005) themselves point out (see also Israelian et al. 2004). It may be, then, that we need to look to the still-increasing samples of DLAs for further clues to the origin of $\mathrm{N}$ in the earliest episodes of star formation.

\section{Concluding Remarks}

It was encouraging to see at this meeting a continuing trend of increasing interaction between stellar and extragalactic astronomers. The former can teach us (the latter) a great deal in our common quest to unravel the physical processes that led to the formation of the first stars, the assembly of galaxies and their evolution to the present time. It is also clear from even this brief review that the tremendous progress we have witnessed in the last five years has only just started us on many paths of enquiry. Returning to the individual topics discussed, I would single out the following as areas where we will be focusing our efforts in the years ahead:

1. The different metallicity diagnostics - from massive stars, $\mathrm{H}$ II regions, and the more widespread interstellar medium - need to be brought together, particularly at the high metallicity end of the scale. Do we understand why we have yet to find clear examples of supersolar metallicities at high redshifts, if the most luminous galaxies we observe then are the progenitors of today's massive ellipticals, as suggested by their masses, clustering and other properties?

2. What are the metallicities and other physical characteristics of star-forming galaxies at the faint end of the luminosity function? How do they overlap with other classes of high redshift objects, such as DLAs and the host galaxies of $\gamma$-ray bursts?

3. We have found no convincing evidence so far for departures from a Salpeter slope of the IMF in galaxies at $z=2-3$. Is this also the case at higher redshifts, or is there a redshift where we can begin to see evidence for the top-heavy IMF generally expected to apply to the first stars? And what of stars less massive than $10 \mathrm{M}_{\odot}$ ? The UV spectra described here give us no clues to their numbers, nor to the characteristic stellar mass $M^{*}$ of the star-formation episodes we observe.

4. Determinations of element abundances in different classes of QSO absorbers continue to complement effectively the large body of stellar work in this area. Stellar rotation is now firmly established as an essential ingredient for a meaningful interpretation of this growing body of data. It will be of great interest to see if physically motivated metallicitydependent $\mathrm{C}, \mathrm{N}$, and $\mathrm{O}$ yields can reproduce the relative abundances of these elements measured in DLAs, as well as Galactic stars, when incorporated into appropriate chemical evolution models.

\section{Acknowledgements}

It is a pleasure to acknowledge the major contributions by my collaborators in the various projects described in this review. Sam Rix also kindly offered valuable comments on the manuscript, and Amanda Smith helped with the preparation of some of the figures. I am indebted to the late Bernard Pagel for numerous inspiring conversations over the years. Finally, I should like to thank the organisers of the Symposium for inviting me to take part in this instructive and stimulating meeting on the beautiful island of Kaua'i.

\section{References}

Akerman, C. J., Carigi, L., Nissen, P. E., et al. 2004, A\&SA, 414, 931

Asplund, M., Grevesse, N., \& Sauval, A. J. 2005, in T. G. Barnes III \& F. N. Bash (eds.), 
Cosmic Abundances as Records of Stellar Evolution and Nucleosynthesis (San Francisco: Astronomical Society of the Pacific), ASP Conf. Ser. 336, 25

Brinchmann, J., Pettini, M., \& Charlot, S. 2008, MNRAS, 385, 679

Centurión, M., Molaro, P., Vladilo, G., et al. 2003, A\&AA, 403, 55

Chiappini, C., Hirschi, R., Meynet, G., et al. 2006, A\& A, 449, L27

Chieffi, A. \& Limongi, M. 2002, ApJ, 577, 281

Crowther, P. A., Prinja, R. K., Pettini, M., \& Steidel, C. C. 2006, MNRAS, 368, 895

de Mello, D. F., Daddi, E., Renzini, A., et al. 2004, ApJ, 608, L29

Erb, D. K., Shapley, A. E., Pettini, M., et al. 2006, ApJ, 644, 813

Henry, R. B. C., Edmunds, M. G., \& Köppen, J. 2000, ApJ, 541, 660

Hirschi, R., Chiappini, C., Meynet, G., et al. 2007, in: R.J. Stancliffe, G. Houdek, R. G. Martin \& C. A. Tout (eds.), Unsolved Problems in Stellar Physics, (New York: AIP) AIP Conf Proc. 948, 397

Hobbs, L. M., York, D. G., \& Oegerle, W. 1982, ApJ, 252, L21

Israelian, G., Ecuvillon, A., Rebolo, R., et al. 2004, A\&A, 421, 649

Jimenez, R. \& Haiman, Z. 2006, Nature, 440, 501

Leitherer, C., Leão, J. R. S., Heckman, T. M., et al. 2001, ApJ, 550, 724

Pauldrach, A. W. A., Hoffmann, T. L., \& Lennon, M. 2001, A\& A, 375, 161

Pettini, M. 2006, in: V. LeBrun, A. Mazure, S. Arnouts \& D. Burgarella (eds.), The Fabulous Destiny of Galaxies: Bridging Past and Present (Paris: Frontier Group), 319

Pettini, M., Lipman, K., \& Hunstead, R. W. 1995, ApJ, 451, 100

Pettini, M. \& Pagel, B. E. J. 2004, MNRAS, 348, L59

Pettini, M., Rix, S. A., Steidel, C. C., et al. 2002, ApJ, 569, 742

Pettini, M., Zych, B. J., Steidel, C. C., \& Chaffee, F. H. 2008, MNRAS, 385, 2011

Pritzl, B. J., Venn, K. A., \& Irwin, M. 2005, AJ, 130, 2140

Prochaska, J. X., Herbert-Fort, S., \& Wolfe, A. M. 2005, ApJ, 635, 123

Reddy, N. A., Steidel, C. C., Pettini, M., et al. 2008, ApJS, 175, 48

Rix, S. A., Pettini, M., Leitherer, C., et al. 2004, ApJ, 615, 98

Shapley, A. E., Steidel, C. C., Adelberger, K. L., et al. 2001, ApJ, 562, 95

Shapley, A. E., Steidel, C. C., Pettini, M., \& Adelberger, K. L. 2003, ApJ, 588, 65

Spite, M., Cayrel, R., Plez, B. et al. 2005, A\& A, 430, 655

Steidel, C. C., Shapley, A. E., Pettini, M., et al., 2004, ApJ, 604, 534

Teplitz, H. I., McLean, I. S., Becklin, E. E., et al., 2000, ApJ, 533, L65

\section{Discussion}

LEITHERER: The galaxies you discussed are UV/optically selected. Another significant component of the star formation census at redshifts $z=2-3$ are sub-mm bright galaxies detected with SCUBA. Could they have higher metallicities than LBGs?

Pettini: This is an interesting possibility, since SCUBA sources are often touted as being the most massive star-forming galaxies at these redshifts. However, the short answer seems to be that their metallicities are no higher than solar, and comparable to those of the most massive LBGs. Of course, the fact that SCUBA galaxies are often very faint at rest-frame UV wavelengths, and the preponderance of AGN amongst them, make this conclusion still rather tentative. However, the work by the Durham group, for example (Swinbank et al. 2004, ApJ, 617, 64), has shown that the $[\mathrm{N} \mathrm{II}] / \mathrm{H} \alpha$ ratio of composite spectra of SCUBA galaxies is indicative of slightly sub-solar abundances, consistent with the strengths of the UV stellar features. If galaxies with clearly supersolar abundances exist at high $z$, we have yet to find them. Part of the problem might well be that in most cases we only obtain a luminosity-weighted, single average value of $Z$ for a given galaxy, with no information on any internal metallicity gradient which may be in place (but see Förster Schreiber et al. 2006, ApJ, 645, 1062). 
Crowther: In Hadfield \& Crowther (2006) we found that Starburst99 with the Geneva or Padova stellar evolution tracks underestimates the ratio of Wolf-Rayet to O-type stars by about a factor of two, when compared with observations of starburst clusters in nearby galaxies whose metallicities are similar to that of the Large Magellanic Cloud. If we were to account for this factor of two, then the metallicity of the $z=3$ LBGs implied by the equivalent width of the He II $\lambda 1640$ emission line measured in the Shapley et al. (2003) composite would fall from solar to near-LMC values (see Figure 8).

PetTini: Thank you for pointing this out. An LMC-type metallicity (somewhere between half and one-third solar) is in fact more likely for the composite of hundreds of LBGs constructed by Shapley et al., based on other abundance indicators. There may well be a number of such systematic offsets in the calibration of the luminosity of the He II $\lambda 1640$ emission line with metallicity. That's why I think that the studies of Wolf-Rayet stars in external galaxies which you and your collaborators are leading are so important for the correct interpretation of the spectra of high- $z$ galaxies.

BurbidGe: You have shown some very beautiful results. To obtain them you use the brightest sources. Do they tend to be average sources, i.e. typical objects in the redshift range, or are they to some extent anomalous in the sense that they are much brighter and easier to observe than average objects at the same redshift?

Pettini: The observations I have presented refer to galaxies spanning a range of luminosities, from the bright end of the luminosity function down to one-to-two magnitudes (depending on redshift) below the fiducial luminosity $L^{*}$. Whether they are typical of the galaxy population at these redshifts depends on your definition of 'typical'. I would contend that they are not just the 'tip of the iceberg', as it is sometimes claimed. Of course, it would be of great interest to extend the work I have described to the more numerous galaxies at the faint end of the luminosity function. Such observations will have to wait until the advent of the next generation of giant optical-infrared telescopes, although we may get glimpses ahead of time aided by the boost of gravitational lensing.

MeYnet: Primary N production is accompanied by the production of primary ${ }^{13} \mathrm{C}$. Thus, if signs of primary nitrogen are visible, one may expect low values of the ${ }^{12} \mathrm{C} /{ }^{13} \mathrm{C}$ ratio in the interstellar medium and at the surfaces of non-evolved stars. Is there any hope to check the ${ }^{12} \mathrm{C} /{ }^{13} \mathrm{C}$ values in DLAs?

Pettini: There has already been one attempt to do so (Levshakov et al. 2006, A\&A, 447 , L21), although in that case the DLA considered was not among the most metalpoor, so that the lack of significant ${ }^{13} \mathrm{C}$ enhancement deduced by those authors may not be surprising. In general, this is a very difficult measurement to perform, because the isotope shifts involved are smaller than other sources of line broadening, even in the DLAs which are kinematically very quiescent, such as the examples I showed in my talk. I am not saying that it is an impossible measurement to perform, but it would require a great deal of careful preparatory work (to select the most likely cases), long exposure times, and a very rigorous analysis of the spectra. 